\title{
NONLINEAR OPERATIONS AND THE SOLUTION OF INTEGRAL EQUATIONS ${ }^{1}$
}

BY

\author{
JON C. HELTON
}

\begin{abstract}
The letters $S, G$ and $H$ denote a linearly ordered set, a normed complete Abelian group with zero element 0 , and the set of functions from $G$ to $G$ that map 0 into 0 , respectively. In addition, if $V \in H$ and there exists an additive function $\alpha$ from $S \times S$ to the nonnegative numbers such that $\|V(x, y) P-V(x, y) Q\|<\alpha(x, y)\|P-Q\|$ for each $\{x, y, P, Q\}$ in $S \times S \times G \times G$, then $V \in O S$ only if $\int_{x}^{y} V P$ exists for each $\{x, y, P\}$ in $S \times S \times G$, and $V \in O Q$ only if ${ }_{x} \Pi^{y}(1+V) P$ exists for each $\{x, y, P\}$ in $S \times S \times G$. It is established that $V \in O S$ if, and only if, $V \in O \Phi$. Then, this relationship is used in the solution of integral equations of the form $f(x)=h(x)+\int_{c}^{x}[U(u, v) f(u)+V(u, v) f(v)]$, where $U$ and $V$ are in $O \delta$. This research extends known results in that requirements pertaining to the additivity of $U$ and $V$ are weakened.
\end{abstract}

1. Introduction. Product integration is used to obtain solutions for linear and nonlinear integral equations of the form

$$
f(x)=h(x)+\int_{x}^{c} V(u, v) f(v) .
$$

In the linear setting, $f$ and $h$ are functions from $S$ to $N$ and $V$ is a function from $S \times S$ to $N$, where $S$ denotes a linearly ordered set and $N$ denotes a normed complete ring. In the nonlinear setting, $f$ and $h$ are functions from $S$ to $G$ and $V$ is a function from $S \times S$ to the set of functions mapping $G$ into $G$, where $G$ denotes a normed complete Abelian group.

We first outline a sequence of results on the solution of the linear form of the integral equation in (1). J. S. Mac Nerney [10] introduces classes $\theta \mathbb{Q}$ and $O \Re$ of functions from $S \times S$ to $N$ such that $V \in \mathcal{O Q}$ if, and only if, $V$ is additive and has bounded variation, and $W \in \mathcal{O} \Re$ if, and only if, $W$ is multiplicative and $W-1$ has bounded variation. He then establishes a one-to-one correspondence between the elements of $O Q$ and $O \mathscr{R}$ and uses this correspondence to obtain solutions for integral equations of the form given in (1), where $V$ is assumed to be an element of $\mathcal{O Q}$ :

Received by the editors June 22, 1976 and, in revised form, October 1, 1976.

AMS (MOS) subject classifications (1970). Primary 45N05; Secondary 47H15.

Key words and phrases. Sum integral, product integral, subdivision-refinement integral, nonlinear integral equation.

'This research was supported in part by a grant from Arizona State University.

- American Mathematical Society 1978 
The preceding results by Mac Nerney are extended by B. W. Helton [2]. He introduces classes $O A^{0}$ and $O M^{0}$ of functions from $S \times S$ to $N$ such that $V \in O A^{0}$ if, and only if, $\int_{a}^{b} V$ exists and $\int_{a}^{b}\left|V-\int V\right|$ exists and is equal to zero for each $\{a, b\}$ in $S \times S$, and $V \in O M^{0}$ if, and only if, ${ }_{a} \Pi^{b}(1+V)$ exists and $\int_{a}^{b}|1+V-\Pi(1+V)|$ exists and is equal to zero for each $\{a, b\}$ in $S \times S$. In this treatment, the existence of

$$
\int_{a}^{b}\left|V-\int V\right|=0 \text { and } \int_{a}^{b}|1+V-\Pi(1+V)|=0
$$

is used to replace the additive and multiplicative conditions required by Mac Nerney. Helton establishes that, if $V$ has bounded variation, then $V \in O A^{0}$ if, and only if, $V \in O M^{0}$, and uses this correspondence to obtain solutions for integral equations of the form

$$
f(x)=h(x)+\int_{c}^{x}[U(u, v) f(u)+V(u, v) f(v)],
$$

where $U$ and $V$ are assumed to be elements of $O A^{0}$ having bounded variation. These results are extensions of the results by Mac Nerney since $O Q$ is a proper subset of the set to which $V$ belongs if, and only if, $V$ has bounded variation and is in $O A^{0}$.

In turn, the results by B. W. Helton are extended by J. C. Helton [4]-[6]. Here, requirements pertaining to the existence of the integrals in (2) are dropped. It is established that, if $V$ has bounded variation, then $\int_{a}^{b} V$ exists for each $\{a, b\}$ in $S \times S$ if, and only if, $\Pi_{a} \Pi^{b}(1+V)$ exists for each $\{a, b\}$ in $S \times S$, and this correspondence is used to obtain solutions for integral equations of the form given in (3), where $U$ and $V$ are assumed to be integrable functions of bounded variation. These results are extensions of the results by B. W. Helton since the set of all functions in $O A^{0}$ with bounded variation is a proper subset of the set to which $V$ belongs if, and only if, $V$ has bounded variation and $\int_{a}^{b} V$ exists for each $\{a, b\}$ in $S \times S$. However, it should be noted that $S$ is assumed to be a closed number interval in this development.

We have just outlined a sequence of results on the solution of the linear form of the integral equation in (1). We now provide a similar outline for its nonlinear form. J. S. Mac Nerney [11] generalizes his results on the solution of the linear equation to corresponding results for the nonlinear equation. To do this, he redefines $O Q$ and $O \Re$. Now, $O Q$ and $O \Re$ are sets of functions from $S \times S$ to the set of functions mapping a normed complete Abelian group $G$ into itself (and 0 and into 0 ) such that $V \in O Q$ if, and only if, $V$ is additive and there exists an additive function $\alpha$ from $S \times S$ to the nonnegative numbers such that, if $\{x, y, P, Q\}$ is in $S \times S \times G \times G$, then 


$$
\|V(x, y) P-V(x, y) Q\| \leqslant \alpha(x, y)\|P-Q\|,
$$

and $W \in \mathcal{O} \Re$ if, and only if, $W$ is multiplicative and there exists a multiplicative function $\mu$ from $S \times S$ to the set of numbers not less than one such that, if $\{x, y, P, Q\}$ is in $S \times S \times G \times G$, then

$$
\|[W(x, y)-1] P-[W(x, y)-1] Q\|<[\mu(x, y)-1]\|P-Q\| .
$$

He then establishes a one-to-one correspondence between the elements of $\Theta \mathbb{Q}$ and $\theta \Re$ and uses this correspondence to obtain solutions for integral equations fo the form given in (1), where $V$ is assumed to be an element of the redefined set $\theta \mathbb{Q}$. (REMARK. Unless noted otherwise, any future reference

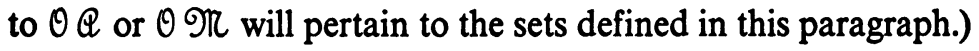

The preceding nonlinear results by J. S. Mac Nerney, as well as the linear results by B. W. Helton, are extended by A. J. Kay [9]. He defines an analog of the integrals in (2) for the nonlinear setting studied by Mac Nerney. In particular, two functions $M$ and $N$ from $S \times S$ to the set of functions mapping $G$ into $G$ are defined to be differentially equivalent if, and only if, there exists a function $k$ from $S \times S$ to the nonnegative numbers such that, if $\{x, y, P\}$ is in $S \times S \times G$, then $\int_{x}^{y} k=0$ and

$$
\|M(x, y) P-N(x, y) P\| \leqslant k(x, y)\|P\| .
$$

Kay establishes that, if $V$ is an element of $O Q \mathcal{Q}$ and $W$ is the corresponding element of $\theta \Re$, then the set of all functions differentially equivalent to $V$ is the same as the set of all functions differentially equivalent to $W-1$, and uses this correspondence to obtain solutions for integral equations of the form given in (3), where $U$ and $V$ are assumed to be functions which are differentially equivalent to elements of $\mathcal{O Q}$. These results extend $J$. S. Mac Nerney's results since $O Q$ is a proper subset of the set of all functions differentially equivalent to $\theta \mathbb{Q}$. Further, the results by B. W. Helton are also extended since the set to which $V$ belongs if, and only if, $V$ has bounded variation and $V$ is in $O A^{0}$, is a proper subset of the set of all functions differentially equivalent to $O Q \mathcal{Q}$.

The purpose of this paper is to extend the results of A. J. Kay by considering a broader class of functions than those differentially equivalent to $O Q$. This extension is analogous to the extension by $\mathbf{J}$. C. Helton for results obtained by B. W. Helton, where requirements involving the integrals in (2) are dropped. In particular, it is established that, if $V$ is a function from $S \times S$ to the set of all functions mapping $G$ into $G$ and there exists a function $\alpha$ from $S \times S$ to the nonnegative numbers such that, if $\{x, y, P, Q\}$ is in $S \times S \times G \times G$, then the inequality in (4) is satisfied, then $\int_{a}^{b} V P$ exists for each $\{a, b, P\}$ in $S \times S \times G$ if, and only if, ${ }_{a} \Pi^{b}(1+V) P$ exists for each $\{a, b, P\}$ in $S \times S \times G$. This correspondence is used to obtain solutions for integral equations of the form given in (3), where $U$ and $V$ are assumed to be 
functions such that, if $\{x, y, P, Q\}$ is in $S \times S \times G \times G$, then each of $\int_{x}^{y} U P$ and $\int_{x}^{y} V P$ exists and each of $U$ and $V$ satisfies the Lipschitz condition implied by the inequality in (4). These results extend Kay's results since the set of all functions differentially equivalent to $O Q Q$ is a proper subset of the set of all functions satisfying the conditions described at the end of the preceding sentence.

In the foregoing, we have described where the results of this paper stand in regard to other closely related papers on the solution of linear and nonlinear forms of the integral equations in (1) and (3). These relationships are summarized in Table 1.

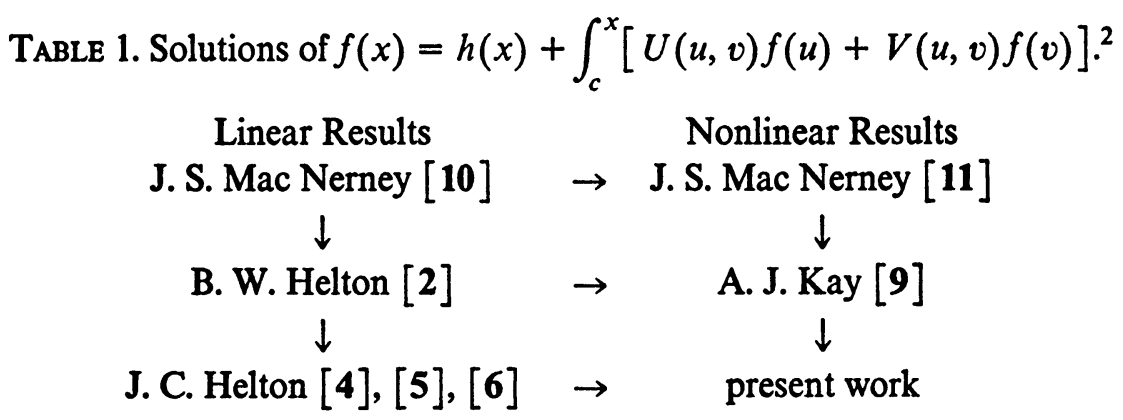

2. Definitions. In the following, $S$ denotes a nondegenerate set with a linear ordering $\theta,\{G,+,\|\cdot\|\}$ denotes a normed complete Abelian group with zero element 0 and $H$ denotes the set of all functions from $G$ to $G$ to which $\{0,0\}$ belongs, with identity function 1 .

If $V$ is a function from $S \times S$ to $G$ and $\{a, b\}$ is in $S \times S$, then the statement that $\int_{a}^{b} V$ exists means there exists an element $L$ of $G$ such that, if $\varepsilon>0$, then there exists a subdivision $D$ of $\{a, b\}$ such that, if $\left\{x_{i}\right\}_{i=0}^{n}$ is a refinement of $D$, then

$$
\left\|L-\sum_{i=1}^{n} V_{i}\right\|<\varepsilon,
$$

where $V_{i}$ denotes $V\left(x_{i-1}, x_{i}\right)$ for $i=1,2, \ldots, n$. If $V$ is a function from $S \times S$ to $H$ and $\{a, b, P\}$ is in $S \times S \times G$, then the statement that ${ }_{a} \Pi^{b}(1+V) P$ exists means there exists an element $L$ of $G$ such that, if $\varepsilon>0$, then there exists a subdivision $D$ of $\{a, b\}$ such that, if $\left\{x_{i}\right\}_{i=0}^{n}$ is a refinement of $D$, then

$$
\left\|L-\prod_{i=1}^{n}\left(1+V_{i}\right) P\right\|<\varepsilon,
$$

\footnotetext{
${ }^{2}$ Only results which pertain to the present study are included in this table.
} 
where $\prod_{i=1}^{n}\left(1+V_{i}\right) P$ denotes the image of $P$ under continued function composition.

If $V$ is a function from $S \times S$ to $H$, then the statement that $V$ has Lipschitz function $\alpha$ means that $\alpha$ is an additive function from $S \times S$ to the nonnegative numbers such that, if $\{x, y, P, Q\}$ is in $S \times S \times G \times G$, then

$$
\|V(x, y) P-V(x, y) Q\| \leqslant \alpha(x, y)\|P-Q\| .
$$

The statement that $V$ satisfies the Lipschitz condition means that such a function $\alpha$ exists.

The symbol $O S$ denotes the set of functions from $S \times S$ to $H$ such that $V$ is in $\mathcal{O S}$ if, and only if, (i) $\int_{a}^{b} V P$ exists for each $\{a, b, P\}$ in $S \times S \times G$, and (ii) $V$ satisfies the Lipschitz condition. The symbol $O \mathscr{P}$ denotes the set of functions from $S \times S$ to $H$ such that $V$ is in $O \mathcal{P}$ if, and only if, (i) ${ }_{a} \Pi^{b}(1+V) P$ exists for each $\{a, b, P\}$ in $S \times S \times G$, and (ii) $V$ satisfies the Lipschitz condition. The representations $O S$ and $\mathcal{O} \odot$ are selected to suggest sum integrable functions and product integrable functions, respectively.

The set of all functions differentially equivalent to Mac Nerney's set $O \mathscr{Q}$ (for the nonlinear setting) is a proper subset of $O S$. With regard to this, the reader is referred to results by W. D. L. Appling [1, Theorem 2, p. 155] and J. C. Helton [3, p. 153].

A function $h$ from $S$ to $G$ has bounded variation if, and only if, there is an additive function $\beta$ from $S \times S$ to the nonnegative numbers such that, if $\{x, y\}$ is in $S \times S$, then

$$
\|h(y)-h(x)\| \leqslant \beta(x, y) .
$$

Further, a function $h$ from $S$ to $G$ is quasi-continuous if, and only if, there exists a sequence $\left\{h_{i}\right\}_{i=1}^{\infty}$ of functions of bounded variation from $S$ to $G$ such that, if $\{x, y\}$ is in $S \times S$, then $\left\{h_{i}\right\}_{i=1}^{\infty}$ converges uniformly to $h$ on $\{x, y\}$. Unless $S$ has the least upper bound property, the preceding definition of quasi-continuous may be requiring more than the existence of right and left limits. For example, if $S=R-\{0\}$ and $h(x)=1 / x$, then $h$ has right and left limits but is not quasi-continuous in the sense just defined.

Certain simplifying notations are now presented. Suppose $\{a, b\}$ is in $S \times S,\left\{x_{i}\right\}_{i=0}^{n}$ is a subdivision of $\{a, b\}, f$ is a function defined on $S$ and $V$ is a function defined on $S \times S$. Then, $f_{i}=f\left(x_{i}\right)$ for $i=0,1, \ldots, n$ and, as noted earlier, $V_{i}=V\left(x_{i-1}, x_{i}\right)$ for $i=1,2, \ldots, n$. Further, if $\left\{x_{i j}\right\}_{j=0}^{n(i)}$ denotes a subdivision of $\left\{x_{i-1}, x_{i}\right\}$ for $i=1,2, \ldots, n$, then $f_{i j}=f\left(x_{i j}\right)$ for $i=$ $1,2, \ldots, n$ and $j=0,1, \ldots, n(i)$, and $V_{i j}=V\left(x_{i j-1}, x_{i j}\right)$ for $i=$ $1,2, \ldots, n$ and $j=1,2, \ldots, n(i)$.

Additional background on the results in this paper and on product integration in general can be obtained in papers by J. C. Helton [6], J. C. Helton and S. Stuckwisch [7], A. J. Kay [9], B. W. Helton [2], J. S. 
Mac Nerney [10], [11] and P. R. Masani [12].

3. Results. We initially establish an existence theorem for sum integrals. This result is useful later in our development.

THEOREM 1. If $f$ and $g$ are quasi-continuous functions from $S$ to $G$ and $V$ is in $O S$, then

and

(i) $\int_{a}^{b} V(u, v)[f(u)+g(v)]$

(ii) $\int_{a}^{b}\left[\int_{u}^{0} V\right][f(u)+g(v)]$ exist and are equal for each $\{a, b\}$ in $S \times S$.

Proof. The existence of the integral in (ii) follows by an argument similar to the one used by J. S. Mac Nerney [11, Lemma 2.2, p. 629] to establish the existence of right integrals of the form $\int_{a}^{b} V(u, v) g(v)$, where $V$ is additive and $g$ is quasi-continuous. We now establish that the integral in (i) exists and is equal to the integral in (ii). Suppose $\{a, b\}$ is in $S \times S$ and $\varepsilon>0$.

It follows from the existence of the integral in (ii) that there exists a subdivision $D_{1}$ of $\{a, b\}$ such that, if $\left\{s_{i}\right\}_{i=0}^{m}$ is a refinement of $D_{1}$, then

$$
\left\|\int_{a}^{b}\left[\int_{u}^{v} V\right][f(u)+g(v)]-\sum_{i=1}^{m}\left[\int_{i} V\right]\left[f_{i-1}+g_{i}\right]\right\|<\frac{\varepsilon}{3},
$$

where $\int_{i} V=\int_{s_{i-1}}^{s_{i}} V$ for $i=1,2, \ldots, m$.

Let $\alpha$ denote a Lipschitz function for $V$. Since $f$ and $g$ are quasi-continuous, there exists a subdivision $D_{2}$ of $\{a, b\}$ such that, if $\left\{s_{i}\right\}_{i=0}^{m}$ is a refinement of $D_{2}$ and $\left\{s_{i j}\right\}_{j=0}^{m(i)}$ is a subdivision of $\left\{s_{i-1}, s_{i}\right\}$ for $i=1,2, \ldots, m$, then

$$
\sum_{i=1}^{m} \sum_{j=1}^{m(i)}\left\|\left(f_{i-1}-f_{i j-1}\right)+\left(g_{i}-g_{i j}\right)\right\| \alpha_{i j}<\varepsilon / 3 .
$$

Let $\left\{s_{i}\right\}_{i=0}^{m}$ denote the subdivision $D_{1} \sqcup D_{2}$ of $\{a, b\}$. For $i=1,2, \ldots, m$, there exists a subdivision $E_{i}$ of $\left\{s_{i-1}, s_{i}\right\}$ such that, if $\left\{s_{i j}\right\}_{j=0}^{m(i)}$ is a refinement of $E_{i}$, then

$$
\left\|\int_{s_{i-1}}^{s_{i}} V\left[f_{i-1}+g_{i}\right]-\sum_{j=1}^{m(i)} V_{i j}\left[f_{i-1}+g_{i}\right]\right\|<\frac{\varepsilon}{3 m} .
$$

Let $D$ denote the subdivision $\sqcup{ }_{i=1}^{m} E_{i}$ of $\{a, b\}$. Further, suppose $\left\{x_{j}\right\}_{j=0}^{n}$ is a refinement of $D,\left\{s_{i j}\right\}_{j=0}^{m(I)}$ is a refinement of $E_{i}$ for $i=1,2, \ldots, m$, and $\left\{x_{j}\right\}_{j=0}^{n}$ is equal to $\sqcup_{i=1}^{m}\left\{s_{i j}\right\}_{j=0}^{m}$. Now, 


$$
\begin{aligned}
\left\|\int_{a}^{b}\left[\int_{u}^{v} V\right][f(u)+g(v)]-\sum_{j=1}^{n} V_{j}\left[f_{j-1}+g_{j}\right]\right\| \\
\quad<\left\|\sum_{i=1}^{m}\left[\int_{i} V\right]\left[f_{i-1}+g_{i}\right]-\sum_{j=1}^{n} V_{j}\left[f_{j-1}+g_{j}\right]\right\|+\varepsilon / 3 \\
\quad \forall\left\|\sum_{i=1}^{m}\left[\int_{i} V\right]\left[f_{i-1}+g_{i}\right]-\sum_{i=1}^{m}\left[\sum_{j=1}^{m(i)} V_{i j}\right]\left[f_{i-1}+g_{i}\right]\right\| \\
\quad+\left\|\sum_{i=1}^{m}\left[\sum_{j=1}^{m(i)} V_{i j}\right]\left[f_{i-1}+g_{i}\right]-\sum_{i=1}^{m} \sum_{j=1}^{m(i)} V_{i j}\left[f_{i j-1}+g_{i j}\right]\right\|+\varepsilon / 3 \\
\quad\left\|\sum_{i=1}^{m}\left[\sum_{j=1}^{m(i)} V_{i j}\right]\left[f_{i-1}+g_{i}\right]-\sum_{i=1}^{m} \sum_{j=1}^{m(i)} V_{i j}\left[f_{i, j-1}+g_{i j}\right]\right\| \\
\quad+m(\varepsilon / 3 m)+\varepsilon / 3 \\
\quad \leqslant \sum_{i=1}^{m} \sum_{j=1}^{m(i)}\left\|\left[f_{i-1}+g_{i}\right]-\left[f_{i j-1}+g_{i j}\right]\right\| \alpha_{i j}+2 \varepsilon / 3 \\
\quad<\varepsilon / 3+2 \varepsilon / 3=\varepsilon .
\end{aligned}
$$

Therefore, the integral in (i) exists and is equal to the integral in (ii). This completes the proof of Theorem 1.

We now establish that, if $V$ is in $\mathcal{O S}$, then $V$ is in $\mathcal{O P}$. Four lemmas are needed.

LEMMA 2.1. If $V$ is in $O S$ and $U(x, y) P=\int_{x}^{y} V P$ for each $\{x, y, P\}$ in $S \times S \times G$, then $U$ is in $0 \mathcal{P}$.

Proof. This lemma follows from a result established by J. S. Mac Nerney [11, Theorem 1.1, p. 624].

LEMMA 2.2. If $V$ is in $\mathcal{O S}$ and $\left\{L_{n}\right\}_{n=0}^{\infty}$ is a sequence of elements of $H$ such that

$$
L_{0}(x, y) P=P
$$

and

$$
L_{n}(x, y) P=P+\int_{x}^{y}\left(\int_{u}^{v} V\right) L_{n-1}(v, y) P
$$

for $n=1,2, \ldots$ and each $\{x, y, P\}$ in $S \times S \times G$, then 


$$
{ }_{x} \Pi^{y}\left(1+\int V\right) P=\lim _{n \rightarrow \infty} L_{n}(x, y) P
$$

for each $\{x, y, P\}$ in $S \times S \times G$.

Proof. This lemma is an adaptation of a result established by $J$. S. Mac Nerney [11, Corollary 2.2, p. 630].

LEMMA 2.3. If $V$ is in $\Theta \mathcal{S}$ and $\left\{L_{n}\right\}_{n=0}^{\infty}$ is a sequence of elements of $H$ such that

$$
L_{0}(x, y) P=P
$$

and

$$
L_{n}(x, y) P=P+\int_{x}^{y} V(u, v) L_{n-1}(v, y) P
$$

for $n=1,2, \ldots$ and each $\{x, y, P\}$ in $S \times S \times G$, then

$$
{ }_{x} \Pi^{y}\left(1+\int V\right) P=\lim _{n \rightarrow \infty} L_{n}(x, y) P
$$

for each $\{x, y, P\}$ in $S \times S \times G$.

Proof. This lemma follows as a corollary to Lemma 2.2 and Theorem 1 since

$$
\int_{x}^{y}\left(\int_{u}^{v} V\right) g(v)=\int_{x}^{y} V(u, v) g(v)
$$

whenever $g$ is a quasi-continuous function from $S$ to $G$ defined on $\{x, y\}$.

LEMMA 2.4. If $V$ is in $O S$ with Lipschitz function $\alpha$ and $\{a, b\}$ is in $S \times S$, then there exists a number $B$ such that, if $w$ is a positive integer, $\varepsilon>0$ and $P$ is in $G$, then there exists a subdivision $D$ of $\{a, b\}$ such that, if $\left\{x_{i}\right\}_{i=0}^{m}$ is a refinement of $D, 1 \leqslant k \leqslant m$, and $\left\{y_{j}\right\}_{j=0}^{n}$ is a refinement of $\left\{x_{i}\right\}_{i=k}^{m}$, then

$$
\left\|\prod_{j=1}^{n}\left(1+V_{j}\right) P-L_{w}\left(x_{k}, b\right) P\right\|<B\|P\| \alpha_{w}\left(x_{k}, b\right)+\varepsilon,
$$

where $L_{w}$ is defined in the statement of Lemma $2.3, \alpha_{1}(x, y)=\alpha(x, y)$ for each subdivision $\{a, x, y, b\}$ of $\{a, b\}$ and

$$
\alpha_{i}(x, y)=\int_{x}^{y} \alpha(u, v) \alpha_{i-1}(v, y)
$$

for $i=2,3, \ldots$ and each subdivision $\{a, x, y, b\}$ of $\{a, b\}$.

Proof. Let $B$ represent a number such that, if $\{a, x, b\}$ is a subdivision of $\{a, b\}$ and $P$ is in $G$, then 


$$
\left\|\prod_{i=1}^{n}\left(1+V_{i}\right) P-P\right\|<B\|P\|
$$

for each subdivision $\left\{x_{i}\right\}_{i=0}^{n}$ of $\{x, b\}$.

The lemma is established by induction on $w$. Suppose $w=1, \varepsilon>0$ and $P$ is in $G$. There exists a subdivision $D$ of $\{a, b\}$ such that, if $\left\{x_{i}\right\}_{i=0}^{m}$ is a refinement of $D, 1 \leqslant k \leqslant m$, and $\left\{y_{j}\right\}_{j=0}^{n}$ is a refinement of $\left\{x_{i}\right\}_{i=k}^{m}$, then

$$
\left\|\int_{x_{k}}^{b} V P-\sum_{j=1}^{n} V_{j} P\right\|<\varepsilon .
$$

Suppose $\left\{x_{i}\right\}_{i=0}^{m}$ is a refinement of $D, 1<k<m$, and $\left\{y_{j}\right\}_{j=0}^{n}$ is a refinement of $\left\{x_{i}\right\}_{i=k}^{m}$. Now,

$$
\begin{aligned}
\| \prod_{j=1}^{n}(1 & \left.+V_{j}\right) P-L_{1}\left(x_{k}, b\right) P \| \\
& =\left\|\left\{P+\sum_{j=1}^{n} V_{j} \prod_{q=j+1}^{n}\left(1+V_{q}\right) P\right\}-\left\{P+\int_{x_{k}}^{b} V P\right\}\right\| \\
& <\left\|\sum_{j=1}^{n} V_{j} \prod_{q=j+1}^{n}\left(1+V_{q}\right) P-\sum_{j=1}^{n} V_{j} P\right\|+\varepsilon \\
& \leqslant \sum_{j=1}^{n}\left\|\prod_{q=j+1}^{n}\left(1+V_{q}\right) P-P\right\|_{\alpha_{j}}+\varepsilon \\
& \leqslant B\|P\| \sum_{j=1}^{n} \alpha_{j}+\varepsilon \\
& =B\|P\| \alpha_{1}\left(x_{k}, b\right)+\varepsilon .
\end{aligned}
$$

Thus, the lemma is established for $w=1$.

The lemma is now assumed to be true for $w$ and established for $w+1$. Suppose $\varepsilon>0$ and $P$ is in $G$. There exists a subdivision $D_{1}$ of $\{a, b\}$ such that, if $\left\{x_{i}\right\}_{i=0}^{m}$ is a refinement of $D_{1}, 1<k<m$, and $\left\{y_{j}\right\}_{j=0}^{n}$ is a refinement of $\left\{x_{i}\right\}_{i=k}^{m}$, then

$$
\left\|\int_{x_{k}}^{b} V(u, v) L_{w}(v, b) P-\sum_{j=1}^{n} V_{j} L_{w}\left(y_{j}, b\right) P\right\|<\frac{\varepsilon}{2}
$$

From the induction hypothesis, there exists a subdivision $D_{2}$ of $\{a, b\}$ such that, if $\left\{x_{i}\right\}_{i=0}^{m}$ is a refinement of $D_{2}, 1 \leqslant k \leqslant m$, and $\left\{y_{j}\right\}_{j=0}^{n}$ is a refinement of $\left\{x_{i}\right\}_{i=k}^{m}$, then 


$$
\left\|\prod_{j=1}^{n}\left(1+V_{j}\right) P-L_{w}\left(x_{k}, b\right) P\right\|<B\|P\| \alpha_{w}\left(x_{k}, b\right)+\varepsilon_{1},
$$

where $\varepsilon_{1}=\varepsilon[2+2 \alpha(a, b)]^{-1}$.

Let $D$ denote the subdivision $D_{1} \sqcup D_{2}$ of $\{a, b\}$. Suppose $\left\{x_{i}\right\}_{i=0}^{m}$ is a refinement of $D, 1<k<m$, and $\left\{y_{j}\right\}_{j=0}^{n}$ is a refinement of $\left\{x_{i}\right\}_{i=k}^{n}$. Now,

$$
\begin{aligned}
\| \prod_{j=1}^{n} & \left(1+V_{j}\right) P-L_{w+1}\left(x_{k}, b\right) P \| \\
& =\left\|\left\{P+\sum_{j=1}^{n} V_{j} \prod_{q=j+1}^{n}\left(1+V_{q}\right) P\right\}-\left\{P+\int_{x_{k}}^{b} V(u, v) L_{w}(v, b) P\right\}\right\| \\
& <\left\|\sum_{j=1}^{n} V_{j} \prod_{q=j+1}^{n}\left(1+V_{q}\right) P-\sum_{j=1}^{n} V_{j} L_{w}\left(y_{j}, b\right) P\right\|+\varepsilon / 2 \\
& <\sum_{j=1}^{n}\left\|\prod_{q=j+1}^{n}\left(1+V_{q}\right) P-L_{w}\left(y_{j}, b\right) P\right\| \alpha_{j}+\frac{\varepsilon}{2} \\
& <\sum_{j=1}^{n}\left[B\|P\| \alpha_{w}\left(y_{j}, b\right)+\varepsilon_{1}\right] \alpha_{j}+\frac{\varepsilon}{2} \\
& =B\|P\| \sum_{j=1}^{n} \alpha_{j} \alpha_{w}\left(y_{j}, b\right)+\varepsilon_{1} \sum_{j=1}^{n} \alpha_{j}+\frac{\varepsilon}{2} \\
& <B\|P\| \int_{x_{k}}^{b} \alpha(u, v) \alpha_{w}(v, b)+\frac{\varepsilon}{2}+\frac{\varepsilon}{2} \\
& =B\|P\| \alpha_{w+1}\left(x_{k}, b\right)+\varepsilon .
\end{aligned}
$$

Thus, the lemma is established for $w+1$. This completes the proof of Lemma 2.4 .

THEOREM 2. If $V$ is in $\theta \mathcal{S}$, then $V$ is in $\theta \mathscr{P}$ and

$$
{ }_{a} \Pi^{b}(1+V) P={ }_{a} \Pi^{b}\left(1+\int V\right) P
$$

for each $\{a, b, P\}$ in $S \times S \times G$.

Proof. Since $V$ has a Lipschitz function $\alpha$, it is only necessary to establish that the product integral associated with $V$ exists. Let $\{a, b, P\}$ belong to $S \times S \times G$. From Lemma 2.1, we have that ${ }_{a} \Pi^{b}\left(1+\int V\right) P$ exists. In the following, it is established that ${ }_{a} \Pi^{b}(1+V) P$ exists and is equal to this integral. Let $\varepsilon>0$.

Let $B$ denote a number with the properties described in Lemma 2.4. There exists a positive integer $N_{1}$ such that, if $w>N_{1}$, then 


$$
B\|P\| \alpha_{w}(a, b)<\varepsilon / 3 .
$$

Further, it follows from Lemma 2.3 that there exists a positive integer $N_{2}$ such that, if $w>N_{2}$, then

$$
\left\|a \Pi^{b}\left(1+\int V\right) P-L_{w}(a, b) P\right\|<\frac{\varepsilon}{3},
$$

where $L_{w}(a, b)$ is defined in the statement of Lemma 2.3.

Let $w$ be an integer greater than $N_{1}+N_{2}$. It follows from Lemma 2.4 that there exists a subdivision $D$ of $\{a, b\}$ such that, if $\left\{x_{i}\right\}_{i=0}^{n}$ is a refinement of $D$, then

$$
\left\|L_{w}(a, b) P-\prod_{i=1}^{n}\left(1+V_{i}\right) P\right\|<B\|P\| \dot{\alpha}_{w}(a, b)+\frac{\varepsilon}{3} .
$$

Suppose $\left\{x_{i}\right\}_{i=0}^{n}$ is a refinement of $D$. Now,

$$
\begin{aligned}
\| a \Pi^{b}(1+ & \left.\int V\right) P-\prod_{i=1}^{n}\left(1+V_{i}\right) P \| \\
& <\left\|L_{w}(a, b) P-\prod_{i=1}^{n}\left(1+V_{i}\right) P\right\|+\frac{\varepsilon}{3} \\
& <B\|P\| \alpha_{w}(a, b)+\varepsilon / 3+\varepsilon / 3 \\
& <\varepsilon / 3+\varepsilon / 3+\varepsilon / 3=\varepsilon .
\end{aligned}
$$

Therefore, ${ }_{a} \Pi^{b}(1+V) P$ exists and is equal to ${ }_{a} \Pi^{b}\left(1+\int V\right) P$. This completes the proof of Theorem 2.

We now establish that, if $V$ is in $\mathcal{O P}$, then $V$ is in $\Theta \mathcal{S}$. Three lemmas are needed.

LEMMA 3.1. If $V$ is in $\Theta \mathscr{P}$ and

$$
U(x, y) P=\left[{ }_{x} \Pi^{y}(1+V)-1\right] P
$$

for each $\{x, y, P\}$ in $S \times S \times G$, then $U$ is in $\Theta S$.

Proof. This lemma follows from a result established by J. S. Mac Nerney [11, Theorem 1.1, p. 624].

LEMMA 3.2. If $\alpha$ is an additive function from $S \times S$ to the nonnegative numbers, $\{a, b\}$ is in $S \times S$ and $\varepsilon>0$, then there exists a subdivision $D$ of $\{a, b\}$ such that, if $\left\{x_{i}\right\}_{i=0}^{n}$ is a refinement of $D$ and $\left\{x_{i j}\right\}_{j=0}^{n(i)}$ is a subdivision of $\left\{x_{i-1}, x_{i}\right\}$ for $i=1,2, \ldots, n$, then

$$
\sum_{i=1}^{n}\left[\prod_{j=1}^{n(i)}\left(1+\alpha_{i j}\right)-\left(1+\alpha_{i}\right)\right]<\varepsilon .
$$


Proof. This lemma is an adaptation of a result established by J. S. Mac Nerney [10, Theorem 2.3, p. 152].

LEMMA 3.3. If $\left\{V_{i}\right\}_{i=1}^{n}$ is a sequence with values in $H$ and $\left\{\alpha_{i}\right\}_{i=1}^{n}$ is a numerical sequence such that, if $\{P, Q\}$ is in $G \times G$, then

$$
\left\|V_{i} P-V_{i} Q\right\|<\alpha_{i}\|P-Q\|
$$

for $i=1,2, \ldots, n$, then (conclusion) for each $\{P, Q\}$ in $G \times G$,

$$
\begin{gathered}
\left\|\left[\prod_{i=1}^{n}\left(1+V_{i}\right)-1\right] P-\left[\prod_{i=1}^{n}\left(1+V_{i}\right)-1\right] Q\right\| \\
<\left[\prod_{i=1}^{n}\left(1+\alpha_{i}\right)-1\right]\|P-Q\|,
\end{gathered}
$$

and

(ii)

$$
\begin{aligned}
\left\|\left[\prod_{i=1}^{n}\left(1+V_{i}\right)\right] P-\left[1+\sum_{i=1}^{n} V_{i}\right] P\right\| \\
\leqslant\left[\prod_{i=1}^{n}\left(1+\alpha_{i}\right)-\left(1+\sum_{i=1}^{n} \alpha_{i}\right)\right]\|P\| .
\end{aligned}
$$

Proof. This lemma is an adaptation of a result established by J. S. Mac Nerney [11, Lemma 1.1, p. 623].

THEOREM 3. If $V$ is in $\Theta \mathcal{P}$, then $V$ is in $O S$ and

$$
\int_{a}^{b} V P=\int_{a}^{b}[\Pi(1+V)-1] P
$$

for each $\{a, b, P\}$ in $S \times S \times G$.

Proof. Since $V$ has a Lipschitz function $\alpha$, it is only necessary to establish that the sum integral associated with $V$ exists. Let $\{a, b, P\}$ belong to $S \times S \times G$. From Lemma 3.1, we have that $\int_{a}^{b}[\Pi(1+V)-1] P$ exists. In the following, it is established that $\int_{a}^{b} V P$ exists and is equal to this integral. Let $\varepsilon>0$.

It follows from Lemmas 3.1 and 3.2 that there exists a subdivision $E$ of $\{a, b\}$ such that, if $\left\{s_{j}\right\}_{j=0}^{m}$ is a refinement of $E$ and $\left\{s_{j k}\right\}_{k=0}^{m(j)}$ is a subdivision of $\left\{s_{j-1}, s_{j}\right\}$ for $j=1,2, \ldots, m$, then

$$
\left\|\int_{a}^{b}[\Pi(1+V)-1] P-\sum_{j=1}^{m}\left[s_{j-1} \Pi^{s_{j}}(1+V)-1\right] P\right\|<\frac{\varepsilon}{3}
$$

and 


$$
\sum_{j=1}^{m}\left[\prod_{k=1}^{m(j)}\left(1+\alpha_{j k}\right)-\left(1+\alpha_{j}\right)\right]\|P\|<\frac{\varepsilon}{3} .
$$

For convenience, suppose $E=\left\{s_{j}\right\}_{j=0}^{m}$.

For $j=1,2, \ldots, m$, there exists a subdivision $E_{j}$ of $\left\{s_{j-1}, s_{j}\right\}$ such that, if $\left\{s_{j k}\right\}_{k=0}^{m(U)}$ is a refinement of $E_{j}$, then

$$
\left\|\prod_{j-1} \Pi^{s_{j}}(1+V) P-\prod_{k=1}^{m(j)}\left(1+V_{j k}\right) P\right\|<\frac{\varepsilon}{3 m} .
$$

Let $D$ denote the subdivision $\cup_{j=1}^{m} E_{j}$ of $\{a, b\}$. Further, suppose $\left\{x_{i}\right\}_{i=0}^{n}$ is a refinement of $D,\left\{s_{j k}\right\}_{k=0}^{m(j)}$ is a subdivision of $\left\{s_{j-1}, s_{j}\right\}$ for $j=1,2, \ldots, m$ and $\left\{x_{i}\right\}_{i=0}^{n}$ is equal to $\sqcup_{j=1}^{m}\left\{s_{j k}\right\}_{k=0}^{m(j)}$. Now,

$$
\begin{aligned}
\| \int_{a}^{b}[ & \Pi(1+V)-1] P-\sum_{i=1}^{n} V_{i} P \| \\
& <\left\|\sum_{j=1}^{m}\left[s_{j-1} \prod_{j}^{s_{j}}(1+V)-1\right] P-\sum_{i=1}^{n} V_{i} P\right\|+\frac{\varepsilon}{3} \\
& <\left\|\sum_{j=1}^{m}\left[\prod_{k=1}^{m(j)}\left(1+V_{j k}\right)-1\right] P-\sum_{i=1}^{n} V_{i} P\right\|+\frac{\varepsilon}{3}+\frac{\varepsilon}{3} \\
& <\sum_{j=1}^{m}\left\|\prod_{k=1}^{m(j)}\left(1+V_{j k}\right) P-\left(1+\sum_{k=1}^{m(j)} V_{j k}\right) P\right\|+\frac{2 \varepsilon}{3} \\
& \leqslant \sum_{j=1}^{m}\left[\prod_{k=1}^{m(j)}\left(1+\alpha_{j k}\right)-\left[1+\sum_{j=1}^{m(j)} \alpha_{j k}\right]\|P\|+\frac{2 \varepsilon}{3}\right.
\end{aligned}
$$

[Lemma 3.3]

$$
<\varepsilon / 3+2 \varepsilon / 3=\varepsilon .
$$

Therefore, $\int_{a}^{b} V P$ exists and is equal to $\int_{a}^{b}[\Pi(1+V)-1] P$. This completes the proof of Theorem 3 .

The following theorem summarizes the results of Theorems 2 and 3.

THEOREM 4. If $V$ is a function from $S \times S$ to $H$, then the following statements are equivalent:

(1) $V$ is in $O S$, and

(2) $V$ is in $\Theta \mathscr{P}$. 
Proof. This theorem follows as a corollary to Theorems 2 and 3.

The solution of the homogeneous integral equation

$$
f(x)=P+\int_{c}^{x}[U(u, v) f(u)+V(u, v) f(v)]
$$

is now obtained. Four lemmas are needed.

LEMMA 5.1. If $b<1, U$ and $V$ are functions in $\theta \delta$ with Lipschitz functions $\alpha$ and $\beta$, respectively, and $\beta<b$, then ${ }_{x} \Pi^{y}\left(1-\int V\right)^{-1}\left(1+\int U\right) P$ exists for each $\{x, y, P\}$ in $S \times S \times G$.

Proof. This lemma is an adaptation of a result established by A. J. Kay [9, Theorem 3.2, p. 209].

REMARK. For a discussion of inverses of the form $(1-V)^{-1}$, the reader is referred to papers by J. W. Neuberger [13] and J. V. Herod [8].

LEMMA 5.2. If $b<1, U$ and $V$ are in $O S$ with Lipschitz functions $\alpha$ and $\beta$, respectively, $\beta<b,\{x, y, P\}$ is in $S \times S \times G$ and $\varepsilon>0$, then there exists $a$ subdivision $D$ of $\{x, y\}$ such that, if $\left\{x_{i}\right\}_{i=0}^{n}$ is a refinement of $D$ and $1 \leqslant i<n$, then

$$
\left\|\left(1-\int_{i} V\right)^{-1}\left(1+\int_{i} U\right) P-\left(1-V_{i}\right)^{-1}\left(1+U_{i}\right) P\right\|<\varepsilon .
$$

Proof. This lemma follows from the integrability of $U$ and $V$ and certain inequalities given by J. V. Herod [8, inequalities (1) and (3), p. 188].

LEMMA 5.3. If $b<1, U$ and $V$ are in $\theta$ S with Lipschitz functions $\alpha$ and $\beta$, respectively, and $\beta<b$, then

(i) ${ }_{x} \Pi^{y}(1-V)^{-1}(1+U) P$

exists and is equal to

(ii) ${ }_{x} \Pi^{y}\left(1-\int V\right)^{-1}\left(1+\int U\right) P$ for each $\{x, y, P\}$ in $S \times S \times G$.

Proof. The existence of the integral in (ii) follows from Lemma 5.1. Let $K$ and $L$ denote elements of $H$ such that

$$
K=U+V(1-V)^{-1}(1+U)
$$

and

$$
L=\int U+\int V\left(1-\int V\right)^{-1}\left(1+\int U\right)
$$

By applying the identity

$$
(1-B)^{-1}(1+A)=1+A+B(1-B)^{-1}(1+A),
$$

we have that ${ }_{x} \Pi^{y}(1+L) P$ is equal to the integral in (ii). For each $\{x, y, P\}$ 
in $S \times S \times G$, it follows from Theorem 3 that $\int_{x}^{y} L P$ exists and from Theorem 2 that

$$
{ }_{x} \Pi^{y}\left(1+\int L\right) P={ }_{x} \Pi^{y}(1+L) P .
$$

For each $\{x, y, P\}$ in $S \times S \times G$, it follows by using Theorem 1 that

$$
\int_{x}^{y} L P=\int_{x}^{y}\left[U+V\left(1-\int V\right)^{-1}\left(1+\int U\right)\right] P,
$$

and hence it follows by using Lemma 3.2 that $\int_{x}^{y} K P$ exists and that

$$
\int_{x}^{y} K P=\int_{x}^{y} L P \text {. }
$$

Further, for each $\{x, y, P\}$ in $S \times S \times G$, it follows by using Theorem 2 that ${ }_{x} \Pi^{y}(1+K) P$ exists and that

$$
{ }_{x} \Pi^{y}(1+K) P={ }_{x} \Pi^{y}\left(1+\int K\right) P,
$$

and it also follows from the identity in (1) that ${ }_{x} \Pi^{y}(1+K) P$ is equal to the integral in (i).

By using the equalities in (2), (3) and (4), we have

$$
{ }_{x} \Pi^{y}(1+K) P={ }_{x} \Pi^{y}(1+L) P
$$

for each $\{x, y, P\}$ in $S \times S \times G$. Therefore, since ${ }_{x} \Pi^{y}(1+K) P$ is equal to the integral in (i) and ${ }_{x} \Pi^{y}(1+L) P$ is equal to the integral in (ii), the desired equality is established. This completes the proof of Lemma 5.3.

LEMMA 5.4. If $b<1,\{c, P\}$ is in $S \times G, f$ is a function from $S$ to $G, U$ and $V$ are functions in $\mathcal{Q} Q$ with Lipschitz functions $\alpha$ and $\beta$, respectively, $\beta<b$, and $U^{\prime}(y, x) Q$ and $V^{\prime}(y, x) Q$ are equal to $U(x, y) Q$ and $V(x, y) Q$, respectively, for each $\{x, y, Q\}$ in $S \times S \times G$, then the two statements in the conclusion of Theorem 5 are equivalent.

Proof. This lemma is an adaptation of a result established by A. J. Kay [9, Theorem 4.3, p. 218].

THEOREM 5. If $b<1,\{c, P\}$ is in $S \times G, f$ is a function from $S$ to $G, U$ and $V$ are functions in $\Theta \mathcal{S}$ with Lipschitz functions $\alpha$ and $\beta$, respectively, $\beta<b$, and $U^{\prime}(y, x) Q$ and $V^{\prime}(y, x) Q$ are equal to $U(x, y) Q$ and $V(x, y) Q$, respectively, for each $\{x, y, Q\}$ in $S \times S \times G$, then the following statements are equivalent:

(1) f has bounded variation and

$$
f(x)=P+\int_{c}^{x}[U(u, v) f(u)+V(u, v) f(v)]
$$


for each $x$ in $S$, and

$$
f(x)={ }_{x} \Pi^{c}\left(1-V^{\prime}\right)^{-1}\left(1+U^{\prime}\right) P
$$

for each $x$ in $S$.

Proof. $[(1) \rightarrow(2)]$. Suppose (1) is true. Then, by employing Theorem 1 , we have that

$$
\begin{aligned}
f(x) & =P+\int_{c}^{x}[U(u, v) f(u)+V(u, v) f(v)] \\
& =P+\int_{c}^{x}\left\{\left[\int_{u}^{v} U\right] f(u)+\left[\int_{u}^{v} V\right] f(v)\right\}
\end{aligned}
$$

for each $x$ in $S$. Now, since $\int U$ and $\int V$ satisfy the hypothesis of Lemma 5.4, we have that

$$
f(x)={ }_{x} \Pi^{c}\left[1-\left(\int V\right)^{\prime}\right]^{-1}\left[1+\left(\int U\right)^{\prime}\right] P
$$

for each $x$ in $S$, where $\left(\int_{v}^{u} V\right)^{\prime} Q$ and $\left(\int_{v}^{u} U\right)^{\prime} Q$ are equal to $\int_{u}^{v} V Q$ and $\int_{u}^{v} U Q$, respectively, for each $\{u, v, Q\}$ in $S \times S \times G$. Thus, by applying Lemma 5.3, we have that

$$
f(x)={ }_{x}^{c}\left(1-V^{\prime}\right)^{-1}\left(1+U^{\prime}\right) P
$$

for each $x$ in $S$. Therefore, (1) implies (2).

Proof. [(2) $\rightarrow(1)]$. Suppose (2) is true. Then, by applying Lemma 5.3, we have that

$$
f(x)={ }_{x} \Pi^{c}\left[1-\left(\int V\right)^{\prime}\right]^{-1}\left[1+\left(\int U\right)^{\prime}\right] P
$$

for each $x$ in $S$, where $\left(\int V\right)^{\prime}$ and $\left(\int U\right)^{\prime}$ are defined as before. Thus, by applying Lemma 5.4, we have that

$$
\begin{aligned}
f(x) & =P+\int_{c}^{x}\left\{\left[\int_{u}^{v} U\right] f(u)+\left[\int_{u}^{v} V\right] f(v)\right\} \\
& =P+\int_{c}^{x}\{U(u, v) f(u)+V(u, v) f(v)\},
\end{aligned}
$$

where the second equality follows from Theorem 1 . The bounded variation of $f$ follows readily. Therefore, (2) implies (1). This completes the proof of Theorem 5.

In the preceding, we have restricted our study to interval functions in $H$. This restriction is now relaxed by considering a new class $H^{*}$ of functions such that, if $V$ is a function from $S \times S$ to the set of functions mapping $G$ 
into $G$, then $V$ is in $H^{*}$ if, and only if, there exists an additive function $\gamma$ from $S \times S$ to the nonnegative numbers such that, if $\{x, y\}$ is in $S \times S$, then $\int_{x}^{y} V 0$ exists and

$$
\|V(x, y) 0\| \leqslant \gamma(x, y) .
$$

By introducing $H^{*}$, we are weakening the restraint in the definition of $H$ that functions must map 0 into 0 . Let $O \mathcal{S}^{*}$ and $O \mathcal{P}^{*}$ be defined with respect to $H^{*}$ as $\Theta \mathcal{S}$ and $\Theta \odot$ are defined with respect to $H$. The following theorems follow as corollaries to Theorems 1,4 and 5 , respectively, by using a technique presented by J. S. Mac Nerney [11, Remark, p. 637].

THEOREM 6. If $f$ and $g$ are quasi-continuous functions from $S$ to $G$ and $V$ is in $O \mathcal{S}^{*}$, then $\int_{a}^{b} V(u, v)[f(u)+g(v)]$ exists for each $\{a, b\}$ in $S \times S$.

THeOREM 7. If $V$ is a function from $S \times S$ to $H^{*}$, then the following statements are equivalent:

(1) $V$ is in $\mathrm{OS}^{*}$, and

(2) $V$ is in $\theta$ P*.

THEOREM 8. If $b<1,\{c, P\}$ is in $S \times G, f$ is a function from $S$ to $G, U$ and $V$ are functions in $0 \mathcal{S}^{*}$ with Lipschitz functions $\alpha$ and $\beta$, respectively, each of $\beta$ and $\|V 0\|$ is less than $b$, and $U^{\prime}(y, x) Q$ and $V^{\prime}(y, x) Q$ are equal to $U(x, y) Q$ and $V(x, y) Q$, respectively, for each $\{x, y, Q\}$ in $S \times S \times G$, then the following statements are equivalent:

(1) $f$ has bounded variation and

$$
f(x)=P+\int_{c}^{x}[U(u, v) f(u)+V(u, v) f(v)]
$$

for each $x$ in $S$, and

$$
f(x)={ }_{x} \Pi^{c}\left(1-V^{\prime}\right)^{-1}\left(1+U^{\prime}\right) P
$$

for each $x$ in $S$.

Now that Theorem 8 is available, it is easy to solve the nonhomogeneous integral equation

$$
f(x)=h(x)+\int_{c}^{x}[U(u, v) f(u)+V(u, v) f(v)],
$$

where $h$ is a function of bounded variation from $S$ to $G$.

THEOREM 9. If $b<1, c$ is in $S, f$ and $h$ are functions from $S$ to $G, h$ has bounded variation, $U$ and $V$ are functions in $\Theta \mathcal{S}^{*}$ with Lipschitz functions $\alpha$ and $\beta$, respectively, each of $\beta$ and $\|V 0\|$ is less than $b, T(x, y) Q=U(x, y) Q+$ $\int_{x}^{y} d h$ for each $\{x, y, Q\}$ in $S \times S \times G$, and $T^{\prime}(y, x) Q$ and $V^{\prime}(y, x) Q$ are equal to $T(x, y) Q$ and $V(x, y) Q$, respectively, for each $\{x, y, Q\}$ in $S \times S \times$ 
$G$, then the following statements are equivalent:

(1) $f$ has bounded variation and

$$
f(x)=h(x)+\int_{c}^{x}[U(u, v) f(u)+V(u, v) f(v)]
$$

for each $x$ in $S$, and

$$
f(x)={ }_{x} \Pi^{c}\left(1-V^{\prime}\right)^{-1}\left(1+T^{\prime}\right) h(c)
$$

for each $x$ in $S$.

Proof. The integral equation in (1) can be rewritten as

$$
f(x)=h(c)+\int_{c}^{x}[T(u, v) f(u)+V(u, v) f(v)] .
$$

Then the theorem follows as a corollary to Theorem 8 .

\section{BIBLIOGRAPHY}

1. W. D. L. Appling, Interval functions and real Hilbert spaces, Rend. Circ. Mat. Palermo (2) 11 (1962), 154-156. MR 27 \# 4040.

2. B. W. Helton, Integral equations and product integrals, Pacific J. Math. 16 (1966), 297-322. MR 32 \#6167.

3. J. C. Helton, An existence theorem for sum and product integrals, Proc. Amer. Math. Soc. 39 (1973), 149-154. MR 47 \#5596.

4. ___ Mutual existence of sum and product integrals, Pacific J. Math. 56 (1975), 495-516.

5. , Product integrals and the solution of integral equations, Pacific J. Math. 58 (1975), 87-103. MR 52 \#6341.

6. Existence of integrals and the solution of integral equations, Trans. Amer. Math. Soc. 229 (1977), 307-327.

7. J. C. Helton and S. Stuckwisch, Numerical approximation of product integrals, J. Math. Anal. Appl. 56 (1976), 410-437.

8. J. V. Herod, Coalescence of solutions for nonlinear Stieltjes equations, J. Reine Angew. Math. 252 (1972), 187-194. MR 45 \#996.

9. A. J. Kay, Nonlinear integral equations and product integrals, Pacific J. Math. 60 (1975), 203-222.

10. J. S. Mac Nerney, Integral equations and semigroups, Illinois J. Math. 7 (1963), 148-173. MR 26 \# 1726.

11. , A nonlinear integral operation, Illinois J. Math. 8 (1964), 621-638. MR 29 \#5082.

12. P. R. Masani, Multiplicative Riemann integration in normed rings, Trans. Amer. Math. Soc. 61 (1947), 147-192. MR 8, 321.

13. J. W. Neuberger, Toward a characterization of the identity component of rings and near-rings of continuous transformations, J. Reine Angew. Math. 238 (1969), 100-104. MR 40 \#3384.

Department of Mathematics, Arizona State University, Tempe, Arizona 85281 